\title{
The Characterization of Linear Polyethylene SRM's 1482, 1483, and 1484 \\ III. Weight-Average Molecular Weights by Light Scattering
}

\author{
Charles C. Han, Peter H. Verdier, and Herman L. Wagner \\ Institute for Materials Research, National Bureau of Standards, Washington, D.C. 20234
}

(October 13, 1977)

\begin{abstract}
Linear polyethylene Standard Reference Materials SRM 1482, 1483, and 1484 are certified for weightaverage molecular weight $M_{w}$. In this paper the experimental procedures employed for the determination of $M_{w}$ for these materials by light scattering are described, and the techniques used to analyze the data and to estimate limits of systematic error are discussed.
\end{abstract}

Key words: Fraction; light scattering; limiting viscosity number; narrow molecular weight distribution; numberaverage molecular weight; polyethylene; Standard Reference Material; weight-average molecular weight.

\section{Introduction}

Standard Reference Materials SRM 1482, 1483, and 1484 are linear polyethylenes with relatively narrow distributions in molecular weight, issued by the National Bureau of Standards. Their general characteristics are described in the first paper of this series [1]. ${ }^{1}$ In the present paper, we describe the determination of their certificate values of weight-average molecular weight $M_{w}$ from measurements of the scattering of light by solutions of the SRM's in l-chloronaphthalene at $135^{\circ} \mathrm{C}$.

The use of light scattering to determine the molecular weight and size of macromolecules in dilute solution is well established [2]. The usual practice is to measure the scattering from the macromolecules in solution relative to that from a material of known scattering power. The measurements reported in this paper, and consequently the certificate values of $M_{w}$, are referred to values published by Coumou [3] for the scattering of light of wavelength $546 \mathrm{~nm}$ from benzene at $23{ }^{\circ} \mathrm{C}$.

The value of the differential refractive index $d n / d c$ is needed to obtain weight-average molecular weights from light scattering data. The differential refractometer employed for this purpose was calibrated with aqueous solutions of sucrose at $25^{\circ} \mathrm{C}$, using values for the differential refractive index for this system published by Norberg and Sundelöf [4].

\section{Experimental}

\subsection{Light Scattering}

\section{a. Apparatus}

A "SOFICA 42,000 Photo Gonio Diffusometer" light scattering photometer was used for scattering measurements. ${ }^{2}$

\footnotetext{
${ }^{1}$ Figures in brackets indicate literature references at the end of this paper.

${ }^{2}$ Certain commercial equipment, instruments, or materials are identified in this paper in order to specify the experimental procedure. In no case does such identification imply recommendation or endorsement by the National Bureau of Standards, nor does it imply that the material or equipment identified is necessarily the best available for the purpose.
}

Light of nominal wavelength $546 \mathrm{~nm}$ was used, polarized with the electric vector of the incident beam normal to the plane containing the incident and scattered wave vectors (vertical polarization). A green filter and a vertical polarizer were placed in the paths of both the incident and the scattered beam. Measurements were made at $135{ }^{\circ} \mathrm{C}$ with the scattering cells immersed in a vat of silicone oil which had been filtered through a glass frit before use.

A glass rod supplied by the photometer manufacturer was used as a working standard. Its scattering relative to that of benzene was measured at room temperature at a scattering angle of $90^{\circ}$, at the wavelength and polarization used for measurements on polyethylene solutions. During the course of the measurements, the optical alignment of the photometer was checked periodically by comparing the scattering signals from solvent at scattering angles of 45 and $135^{\circ}$. The signals were found to differ by less than 1 percent in all cases. The effects of alignment errors of this size on the values obtained for the mean-square radius and the weight-average molecular weight are discussed in sections 3.2 and 4.9 , respectively.

The benzene used for calibrating the photometer was prepared from Certified A.C.S. grade benzene. The starting material was first extracted eight times with concentrated $\mathrm{H}_{2} \mathrm{SO}_{4}$, four times with a 5 percent aqueous solution of $\mathrm{NaOH}$, and four times with distilled water. It was then dried by mixing with anhydrous $\mathrm{CaCl}_{2}$, and fractionally distilled in glass over sodium metal. Finally, it was passed through a column of silica gel and adsorption alumina to adsorb any remaining fluorescent impurities.

\section{b. Preparation of Solutions}

Commercial grade 1-chloronaphthalene was distilled at reduced pressure after removal of residual naphthalene by sublimation, also at reduced pressure. Immediately before use, dissolved air was swept out of the solvent by bubbling oxygen-free nitrogen gas (The Matheson Co., "prepurified grade") through it at room temperature for at least $1 \mathrm{~h}$. Polyethylene solutions were made up by weight, and their 
concentrations were calculated using values of solvent density and partial specific volume at $135{ }^{\circ} \mathrm{C}$ determined pycnometrically in this laboratory. The polyethylene samples were dissolved at $135{ }^{\circ} \mathrm{C}$ with gentle stirring. They were then filtered, as described in section 2.1.3, directly into the lightscattering cells and the scattering recorded. In preliminary experiments, the filters employed showed discoloration and signs of disintegration after $2 \mathrm{~h}$ exposure to 1-chloronaphthalene at $140{ }^{\circ} \mathrm{C}$. Evidence of degradation (discoloration, increased and erratic small-angle scattering) was also found when filtered solutions were kept at $135^{\circ} \mathrm{C}$ for more than $2 \mathrm{~h}$, but no such evidence was found in less than $2 \mathrm{~h}$. The final measurements were therefore scheduled so that the total exposure time of the filters to hot 1-chloronaphthalene was less than $1 / 2 \mathrm{~h}$ in all cases, and so that no more than $2 \mathrm{~h}$ elapsed between the beginning of heating and completion of the measurements of each solution.

\section{c. Measurement Procedure}

Before each use, xylene vapor was allowed to condense on the inside surfaces of the inverted light scattering cells, to remove dust. Polyethylene solutions or solvent were filtered directly into the cells through a hypodermic syringe heated to $135^{\circ} \mathrm{C}$ and fitted with a "Swinney Adapter" filter holder (Millipore Corp., Bedford, Mass.). For measurements on SRM's 1483 and 1484 and their associated solvent readings, solutions and solvents were filtered through $0.22 \mu \mathrm{m} \mathrm{MF}$ Millipore filters (No. GSWPO 2500) made of mixed esters of cellulose. No external pressure was applied during the filtration.

The scattering from SRM 1482 was much smaller than that from the other two polyethylenes, and noise from dirt and dust was a more serious problem. For measurements on this material, the final de-dusting of the scattering cells by condensed xylene vapor was preceded by boiling in xylene, soaking in hot chromic acid, and successive rinsing in tap water, distilled water, and ethanol. Two stacked $0.22 \mu \mathrm{m}$ Millipore filters were used to filter SRM 1482 solutions and solvent into the scattering cells. External pressure of about $0.5 \mathrm{psi}^{*}$ was applied during filtration and adjusted to give a flow rate of about $1 / 2 \mathrm{~mL} / \mathrm{min}$., in order to make the total exposure time of the filter to hot 1-chloronaphthalene less than $1 / 2 \mathrm{~h}$.

For all three SRM's, the filter was first rinsed with the preheated material to be measured (solvent or solution), to remove detergent added during the filter manufacturing process and surface dust. Three rinses of about $2 \mathrm{ml}$ each were then filtered into the scattering cell and discarded. Finally, 8 to $10 \mathrm{ml}$ were filtered into the cell for measurement, and the cell was placed in the photometer and allowed to reach thermal equilibrium.

Measurements of scattered intensity were made at scattering angles from 45 to $135^{\circ}$ in $15^{\circ}$ steps. The scattering signal at $90^{\circ}$ from the glass rod used as a working standard was measured after each solution or solvent measurement. For measurements on SRM 1483, solvent measurements were made in three of the five scattering cells used. The three sets of solvent measurements were averaged, and the average readings used for the analysis of the measurements on all the solutions. For measurements on SRM's 1482 and 1484, solvent measurements were taken in every cell used for

\footnotetext{
* 1 psi equals approximately $7 \times 10^{3} \mathrm{~Pa}$
}

solution measurements, and the solvent scattering intensities obtained for each cell were used for the analysis of measurements on solutions taken with that cell. In addition, each cell was always placed in the photometer with the same angular orientation to minimize the effects of imperfections in the cells.

Seven sets of measurements were made on SRM 1482, five sets on SRM 1483, and six sets on SRM 1484. Each set consisted of measurements on solutions with nominal concentration of: $3,4,5,6.5,8,10$, and $12 \mathrm{~g} / \mathrm{L}$ for SRM 1482; , $3,4.5,6$, and $10 \mathrm{~g} / \mathrm{L}$ for SRM 1483; 0.6, 0.8, 1, 1.3, 1.8, 2.3 , and $3 \mathrm{~g} / \mathrm{L}$ for SRM 1484. For SRM 1483, each set contained one solution at each of the nominal concentrations; some sets for SRM's 1482 and 1484 omitted at most one of the nominal concentrations. Thus, the sets of measurements on SRM 1483 are equivalent, and those for SRM's 1482 and 1484 are nearly so.

\subsection{Differential Refractive Index}

The differential refractometer employed for measurements on SRM's 1482, 1483, and 1484 has been described elsewhere [5]. For the present work, it was modified slightly by the addition of a condensing lens between the mercury lamp source and the slit. The instrument was calibrated with 7 solutions of sucrose, with concentrations from 6.3 to $26 \mathrm{~g} / \mathrm{L}$, at $25{ }^{\circ} \mathrm{C}$. The sucrose solutions were made up by weight, and their concentrations were calculated using the density tables of Schneider et al.[6]. Otherwise, the equipment and measurement techniques employed in this work were identical with those described in [5].

\section{Results}

\subsection{Differential Refractive Index}

The experimental determination of the differential refractive index $d n / d c$ described in detail in [5] consists essentially of the measurement of the displacement $d$ of an optical slit image resulting from the substitution of a solution of concentration $c$ for solvent in one side of a measuring cell. For dilute solutions, $d$ is proportional to $c$; the ratio $k=d / c$ is proportional to $d n / d c$. In practice, $k$ is determined by measuring displacement for several concentrations and fitting the observed displacements to $d=k c$. Calibration is accomplished by determining the value $k_{0}$ for a material of known differential refractive index $(d n / d c)_{0}$; then for substance $i$ we have:

$$
(d n / d c)_{i}=(d n / d c)_{0} k_{i} / k_{0} .
$$

Displacements were measured as a function of concentration for SRM's 1482, 1483, and 1484 at $135{ }^{\circ} \mathrm{C}$ in 1-chloronaphthalene, and for aqueous solutions of sucrose at $25{ }^{\circ} \mathrm{C}$. Table 1 shows the resulting values $k_{i}$ (in arbitrary units), together with the estimates $s_{i}(d)$ and $s\left(k_{i}\right)$ of standard deviation in $d$ and $k$, respectively, and the number of degrees of freedom $F_{i}$, equal in each case to one less than the number of concentrations measured. The values of $s_{i}(d)$ for the four materials are consistent with the physically plausible assumption that they are all estimates of the same quantity, i.e., the inherent imprecision of the measurement of $d$ is independent of the sample. We therefore form a pooled 
TABLE 1. Results of differential refractive index measurements at wavelength in vacuum $546 \mathrm{~nm}$.

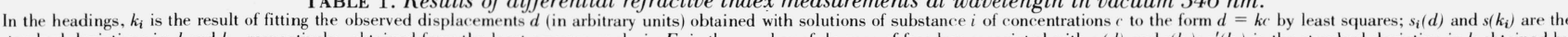

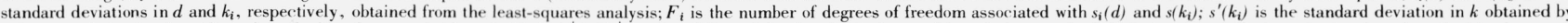

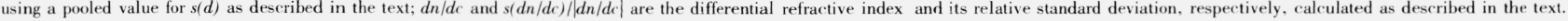

\begin{tabular}{|c|c|c|c|c|c|c|c|c|c|}
\hline Substance & $\begin{array}{c}\text { Range of } \\
\text { concentration } \\
\mathrm{g} / \mathrm{L}\end{array}$ & $k_{i}$ & $s_{i}(d)$ & $s\left(k_{i}\right)$ & $F_{i}$ & $s^{\prime}\left(k_{i}\right)$ & $\begin{array}{c}s^{\prime}\left(k_{i}\right) /\left|k_{i}\right| \\
\text { percent }\end{array}$ & $d n / d c, \mathrm{~mL} / \mathrm{g}$ & $\frac{s(d n / d c)}{|d n / d c|}$, \\
\hline Sucrose $^{a}$ & 6.3 to 26 & 167.46 & 0.00386 & 0.08 & 6 & 0.15 & 0.09 & $0.1429^{b}$ & - \\
\hline SRM $1482^{c}$ & 6.3 to 15 & -228.4 & 0.00878 & 0.38 & 7 & 0.32 & 0.14 & -0.1949 & 0.17 \\
\hline $\mathrm{SRM} 1483^{\mathrm{c}}$ & 5.6 to 13 & -225.6 & 0.00477 & 0.22 & 5 & 0.34 & 0.15 & -0.1925 & 0.17 \\
\hline $\mathrm{SRM} 1484^{\mathrm{c}}$ & 5.6 to 13 & -224.4 & 0.00987 & 0.43 & 6 & 0.32 & 0.14 & -0.1920 & 0.17 \\
\hline Pooled & - & - & 0.00744 & - & 24 & - & - & - & - \\
\hline
\end{tabular}

a Aqueous solutions at $25^{\circ} \mathrm{C}$.

b Value from [4], used for calibration.

c At $135^{\circ} \mathrm{C}$ in 1-chloronaphthalene.

estimate of the variance $v(d)=s^{2}(d)$ as the average of the individual estimates of variance, weighted by the number of degrees of freedom associated with each estimate:

$$
\begin{aligned}
s(d)=[v(d)]^{\frac{1}{2}} & =\left[F^{-1} \sum_{i} F_{i} v_{i}(d)\right]^{\frac{1}{2}} \\
& =\left[F^{-1} \sum_{i} F_{i} s_{i}{ }^{2}(d)\right]^{\frac{1}{2}},
\end{aligned}
$$

where $F=\sum_{i} F_{i}$ and the index $i$ is summed over the four samples. We then use this pooled value to form improved estimates $s^{\prime}\left(k_{i}\right)=s\left(k_{i}\right) s(d) / s_{i}(d)$. Values of $s^{\prime}\left(k_{i}\right)$ and the associated relative standard deviations $s^{\prime}\left(k_{i}\right) /\left|k_{i}\right|$ are shown in table 1 . We then obtain the relative standard deviation in the ratios $k_{i} / k_{0}$, where the subscript zero denotes the measurements on sucrose solutions, as:

$$
s\left(k_{i} / k_{0}\right) /\left|k_{i} / k_{0}\right|=\left\{\left[s^{\prime}\left(k_{i}\right) / k_{i}\right]^{2}+\left[s^{\prime}\left(k_{0}\right) / k_{0}\right]^{2}\right\}^{\frac{1}{2}} .
$$

Finally, we observe that by eq (1), the relative standard deviation $s(d n / d c) /|d n / d c|$ in $d n / d c$ is just $s\left(k_{i} / k_{0}\right) /\left|k_{i} / k_{0}\right|$. Values of $d n / d c$ calculated from the $k$ 's and the literature value for $d n / d c$ of sucrose by eq (1) are shown in table 1 for SRM's 1482, 1483, and 1484, together with the associated values of $s(d n / d c) /|d n / d c|$.

\subsection{Light Scattering}

In principle [2], light scattering data from polymer solutions of concentrations $c$ at scattering angles $\theta$ may be analyzed by fitting $c g / I_{c}$ to a power series in $c$ and $\sin ^{2}(\theta / 2)$, where $g$ is unity for vertical polarization (and $\cos ^{2} \theta$ for horizontal polarization), $I_{c}=\sin \theta[I(\theta, c)-I(\theta, 0)] / I_{G}, I_{G}$ is the scattering signal from the glass working standard at $\theta=$ $90^{\circ}$, and $I(\theta, c)$ is the scattering signal from a solution of concentration $c$ at scattering angle $\theta$ :

$$
c g / I_{c}=\sum_{i j} C_{i j} c^{i} \sin ^{2 j}(\theta / 2) .
$$

In order to use eq (4) for the estimation of molecular parameters, we must first decide how many terms on the right-hand side must be included to provide an adequate fit to the experimental data. The dependence of $c g / I_{c}$ upon $c$ and upon $\sin ^{2}(\theta / 2)$ reflects solute-solute interactions and solute size, respectively. Since both effects increase with increasing molecular weight, we expect the highest molecular-weight material to provide the most sensitive test of adequacy of fit. Accordingly, preliminary scattering data for SRM 1484, with the highest molecular weight of the three SRM's, were first plotted as $c / I_{c}$ versus $\sin ^{2}(\theta / 2)$ at constant concentration and versus $c$ at constant scattering angle, to see whether a linear expansion (i.e., retaining only $C_{00}, C_{01}$, and $C_{10}$ ) would provide an adequate fit. The plots revealed that the linear approximation was clearly inadequate in this case; in particular, the constant-angle plots versus $c$ showed distinct curvature. The effect of including second-order terms in eq (4) $\left(C_{02}, C_{11}\right.$, and $\left.C_{20}\right)$ was therefore investigated by a series of least-squares analyses of the data. Inclusion of $C_{20}$ was found to reduce the residual standard deviation in $c / I_{c}$ by 55 percent, while inclusion of $C_{11}$ resulted in a reduction of only 4 to 10 percent and inclusion of $C_{02}$ increased the residual standard deviations slightly. The number and range of concentrations were not sufficient for inclusion of higher terms in $c\left(C_{30}\right.$, etc. $)$ to be sensible. The final analyses on all three SRM's were therefore carried out including $C_{20}$ but not the other second-order terms:

$$
c / I_{c}=C_{00}+C_{01} \sin ^{2}(\theta / 2)+C_{10} c+C_{20} c^{2} .
$$

The coefficients in eq (5) are related to the weight-average molecular weight $M_{w}$, molecular mean-square radius $R_{G}{ }^{2}$, and second and third virial coefficients $A_{2}$ and $A_{3}$ by $[2,7]$ :

$$
\begin{aligned}
M_{w} & =\left(K^{\prime} C_{00}\right)^{-1}, \\
R_{G}{ }^{2} & =3\left[\lambda_{0} /(4 \pi n)\right]^{2} C_{01} / C_{00} \\
A_{2} & =1 / 2 K^{\prime} C_{10}, \\
A_{3} & =1 / 3 K^{\prime} C_{20}, \\
K^{\prime} & =4 \pi^{2} n_{B}{ }^{2}(d n / d c)^{2} /\left(\lambda_{0}{ }^{4} N_{A} V_{v}{ }^{B} S\right),
\end{aligned}
$$

where:

$\lambda_{0}$ is the wavelength in vacuum of the scattered light, set at $546 \mathrm{~nm}$ in this work,

$n$ and $n_{B}$ are the indices of refraction of the solvent and benzene, taken as 1.586 [8] and 1.503 [3], respectively, 
$d n / d c$ is the differential refractive index of the solution, measured as described in 3.1,

$N_{A}$ is Avogadro's number, taken as $6.022 \times 10^{23} \mathrm{~mol}^{-1}$,

$V_{v}{ }^{B}$ is the Rayleigh ratio for the vertically polarized scattering of vertically polarized light from benzene, used for calibration and obtained as described in the following paragraph, and

$S$ is the measured ratio $I_{G} / I_{B}$ of the scattering signal obtained from the glass working standard to that obtained from benzene.

The "vertical-vertical" Rayleigh ratio $V_{v}$ is related to the Rayleigh ratio $U_{u}$ for the unpolarized scattering of unpolarized light and the depolarization ratio $\rho_{u}$ for unpolarized light by:

$$
V_{v}=U_{u}\left(2-\rho_{u}\right) /\left(1+\rho_{u}\right) .
$$

Using the published [3] values for benzene

$$
\begin{aligned}
& U_{u}=15.8 \times 10^{-6} \mathrm{~cm}^{-1} \\
& \text { and } \rho_{u}=0.41, \text { we obtain } \\
& V_{v}^{B}=17.8 \times 10^{-6} \mathrm{~cm}^{-1} .
\end{aligned}
$$

The uncertainty in $M_{w}$ introduced by the use of this derived Rayleigh ratio is discussed in section 4.4.

As described in section 2.1c, from five to seven sets of measurements were made on each SRM. Each set consisted of scattering measurements on from five to seven solutions, together with solvent measurements, at seven scattering angles. The sets for each SRM are equivalent or nearly so. The data in each set were fitted by least squares to eq (5), and the resulting values of $C_{00}, C_{01}, C_{10}$, and $C_{20}$ were used to calculate $M_{w}, R_{G}{ }^{2}, A_{2}$, and $A_{3}$ using eq (6)-(10). The estimates obtained from each set were then averaged. The resulting mean values and sample standard deviations of the mean are given in table 2 , together with values of the molecular radius of gyration $\left(R_{G}{ }^{2}\right)^{\frac{1}{2}}$, the ratio $A_{3} /\left(M_{w} A_{2}^{2}\right)$, and the number of degrees of freedom (equal in each case to one less than the number of sets).

The values of $M_{w}$ in table 2 may be compared with the number-average molecular weights $M_{n}$ determined by membrane osmometry and reported in paper II of this series [9]. It will be seen that $M_{w}$ is slightly greater than $M_{n}$ for all three SRM's, yielding estimates of the ratio $M_{w} / M_{n}$ in the range 1.1 to 1.2 , consistent with estimates obtained by gel permeation chromatography [1]. The values obtained for the second virial coefficient are in crude but satisfactory agreement with those obtained by membrane osmometry [9] and with literature values for linear polyethylenes in 1-chloronaphthalene [7, 10-12]. In addition, the ratios $A_{3} /\left(M_{w} A_{2}{ }^{2}\right)$ are consistent with the often-cited estimate [13] of $1 / 4$. On the other hand, the values obtained for $R_{G}{ }^{2}$ are clearly absurd, since they indicate that SRM 1482 in solution is more than half again as large as SRM 1483, which has a molecular weight $2 \frac{1}{2}$ times as large as SRM 1482. The obviously large errors in $R_{G}{ }^{2}$ are almost certainly due to a combination of instrumental misalignment and incomplete removal of dust from the solute. For SRM's 1482 and 1483, the intensity at $\theta=45^{\circ}$ is only $2-3$ percent greater than that at $\theta=135^{\circ}$. Consequently an error from either source of 1 percent in the scattering at $45^{\circ}$ relative to that at $135^{\circ}$ would result in errors of 35-100 percent in $R_{G}{ }^{2}$. Clearly, these values for $R_{G}{ }^{2}$ must be treated as no more than very rough estimates.

\section{Systematic Errors}

We now list the likeliest sources of systematic error in the estimates of weight-average molecular weight described in the preceding section, and attempt to set upper limits on their magnitudes. Individual sources of error are discussed in the following subsections; the resulting error-limit estimates are summarized in table 3 , expressed as percent errors applied to $M_{w}$.

According to eq (6), $M_{w}$ is the reciprocal of the product $K^{\prime} C_{00}$, where $K^{\prime}$ is given by eq (10). The effects of errors in the quantities that enter into $K^{\prime}$ are discussed in sections 4.1-4.5. The coefficient $C_{00}$ may be written as the zeroangle, zero-concentration limit of the quantity

$$
\operatorname{cg} \csc \theta /\left[S_{G}(\theta, c)-S_{G}(\theta, 0)\right],
$$

where $S_{G}(\theta, c)$ is the ratio of the signal obtained at scattering angle $\theta$ from a solution of concentration $c$ to that obtained from the glass working standard at the same instrumental gain. Because of the non-ideality of the polarizers placed in the path of the incident and scattered light, the geometric factor $g$ is not strictly unity; the resulting error is discussed in section 4.6. It is clear that systematic errors in $S_{G}(\theta, c)$ and $c$ will affect the value of $C_{00}$ and therefore of $M_{w}$; errors from these sources are discussed in sections 4.7 and 4.8. The assumptions implicit in the use of eq (5), (6) and (10) introduce several possible sources of error; these are discussed in sections 4.9-4.14. Finally, the error limits from all the foregoing sources are combined and summarized in section 4.15 .

\subsection{Index of Refraction of Benzene}

The index of refraction of benzene at $546 \mathrm{~nm}$ and $23{ }^{\circ} \mathrm{C}$ is given in [3] as 1.503. We believe that this value should be good as stated, i.e., to 1 part in 1500 . Since $n_{B}$ appears in $K^{\prime}$ as the square, the resulting relative error in $M_{w}$ is $2 / 1500$ or 0.1 percent.

\subsection{Differential Refractive Index}

Errors in our values for the differential refractive index of the SRM's arise both from possible errors in the literature value for the differential refractive index of sucrose solutions, used for calibration, and from the imprecision of our measurements. We believe that the value of $d n / d c$ for sucrose in [4] is accurate to 1 percent. The relative standard deviation of our own measurements, shown in table 1 , is 0.17 percent for each of the SRM's. We choose the 95 percent confidence limit corresponding to this value as a reasonable error limit. The Student $t$ factor [14] for 24 degrees of freedom and 95 percent confidence limits is 2.064 ; the 95 percent confidence limit is therefore $2.064 \times 0.17$ percent, or 0.35 percent. Finally, since $d n / d c$ appears in $K^{\prime}$ as the square, the resulting errors in $\boldsymbol{M}_{\boldsymbol{w}}$ from the literature sucrose value and our own measurements are 2 percent and 0.7 percent respectively. 
TABLE 2. Molecular parameters obtained by light scattering on solutions of linear polyethylene Standard Reference Materials 1482, 1483, and 1484 in 1-chloronaphthalene at $135^{\circ} \mathrm{C}$. Numbers in parentheses are sample standard deviations of the mean.

\begin{tabular}{|c|c|c|c|c|c|c|c|}
\hline Sample & $\begin{array}{c}\text { Weight-average } \\
\text { molecular } \\
\text { weight, } \\
M_{w}, \mathrm{~g} / \mathrm{mol}\end{array}$ & $\begin{array}{l}\text { Molecular } \\
\text { mean-square } \\
\text { radius, } \\
R_{G}{ }^{2}, \mathrm{~cm}^{2}\end{array}$ & $\begin{array}{c}\text { Molecular } \\
\text { radius of } \\
\text { gyration, } \\
\left(R_{G^{2}}\right)^{1 / 2}, \\
\AA\end{array}$ & 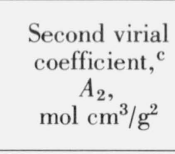 & 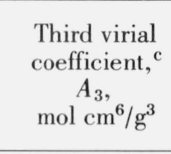 & $\frac{A_{3}}{M_{w^{A_{2}}}{ }^{2}}$ & $\begin{array}{c}\text { Number of } \\
\text { degrees of } \\
\text { freedom }\end{array}$ \\
\hline SRM 1482 & $\begin{array}{c}13.63 \times 10^{3} \\
\left(0.13 \times 10^{3}\right)\end{array}$ & $\begin{array}{r}1.79 \times 10^{-12} \\
\left(0.16 \times 10^{-12}\right.\end{array}$ & $\begin{array}{r}133.7 \\
(6.0)\end{array}$ & $\begin{array}{c}1.59 \times 10^{-3} \\
\left(0.08 \times 10^{-3}\right)\end{array}$ & $\begin{array}{c}1.28 \times 10^{-2} \\
\left(0.37 \times 10^{-2}\right)\end{array}$ & $\begin{array}{c}0.35 \\
(0.19)\end{array}$ & 6 \\
\hline SRM 1483 & $\begin{array}{l}32.1 \times 10^{3} \\
\left(1.6 \times 10^{3}\right)\end{array}$ & $\begin{array}{c}6.75 \times 10^{-13} \\
\left(0.53 \times 10^{-13}\right)\end{array}$ & $\begin{array}{c}82.2 \\
(3.2)\end{array}$ & $\begin{array}{c}1.09 \times 10^{-3} \\
\left(0.10 \times 10^{-3}\right)\end{array}$ & $\begin{array}{c}1.34 \times 10^{-2} \\
\left(0.60 \times 10^{-2}\right)\end{array}$ & $\begin{array}{c}0.63 \\
(0.37)\end{array}$ & 4 \\
\hline SRM 1484 & $\begin{array}{r}119.6 \times 10^{3} \\
\left(2.2 \times 10^{3}\right)\end{array}$ & $\begin{array}{r}4.75 \times 10^{-12} \\
\left(0.10 \times 10^{-12}\right.\end{array}$ & $\begin{array}{r}217.9 \\
(2.4)\end{array}$ & $\begin{array}{c}1.03 \times 10^{-3} \\
\left(0.10 \times 10^{-3}\right)\end{array}$ & $\begin{array}{c}2.8 \times 10^{-2} \\
\left(1.7 \times 10^{-2}\right)\end{array}$ & $\begin{array}{c}0.40 \\
(0.20)\end{array}$ & 5 \\
\hline
\end{tabular}

a Systematic errors in weight-average molecular weight are discusssd in section 4 of the text and summarized in table 3 .

b As discussed in section 3.2 of the text, there is reason to believe that these values are subject to large systematic errors.

c We have not attempted to estimate systematic errors in these quantities.

TABLE 3. Percent errors in $M_{w}$ introduced by assumed errors in measured quantities and approximations

\begin{tabular}{|c|c|c|c|}
\hline \multirow[b]{2}{*}{ Source of Error } & \multicolumn{3}{|c|}{ Error in $M_{w}$, percent } \\
\hline & $\begin{array}{l}\text { SRM } \\
1482\end{array}$ & $\begin{array}{l}\text { SRM } \\
1483\end{array}$ & $\begin{array}{l}\text { SRM } \\
1484\end{array}$ \\
\hline 1. Index of refraction of benzene & 0.1 & 0.1 & 0.1 \\
\hline $\begin{array}{l}\text { 2. Literature value of differential refrac- } \\
\text { tive index of sucrose }\end{array}$ & 2.0 & 2.0 & 2.0 \\
\hline $\begin{array}{l}\text { 3. Measured value of differential refrac- } \\
\text { tive index of SRM's relative to that } \\
\text { of sucrose }\end{array}$ & 0.7 & 0.7 & 0.7 \\
\hline 4. Wavelength of radiation & 0.5 & 0.5 & 0.5 \\
\hline $\begin{array}{l}\text { 5. Rayleigh ratio } V_{v} \text { for benzene, derived } \\
\text { from literature values of } U_{u} \text { and } \rho_{u}\end{array}$ & 10. & 10. & 10. \\
\hline 6. Measured ratios $I_{G} / I_{B}$ & 0.4 & 0.4 & 0.4 \\
\hline 7. Polarizer errors & 0.0 & 0.0 & 0.0 \\
\hline 8. Measured ratios $S_{G}(\theta, c)$ & 0.3 & 0.3 & 0.3 \\
\hline 9. Solvent density & 0.2 & 0.2 & 0.2 \\
\hline 10. Solute weights & 0.3 & 0.4 & 1.2 \\
\hline 11. Reflection correction & 0.0 & 0.0 & 0.0 \\
\hline 12. Instrumental misalignment & 0.7 & 0.7 & 0.7 \\
\hline 13. Refraction correction & 0.4 & 0.4 & 0.4 \\
\hline 14. Anisotropy of polyethylene & 0.1 & 0.1 & 0.0 \\
\hline $\begin{array}{l}\text { 15. Temperature dependence of scattering } \\
\text { of glass working standard }\end{array}$ & 0.4 & 0.4 & 0.4 \\
\hline 16. Cutoff of virial expansion & 0.7 & 1.4 & 0.9 \\
\hline 17. Sum of all the above except (5) & 6.8 & 7.6 & 7.8 \\
\hline $\begin{array}{l}\text { 18. Root-sum-square of all the above ex- } \\
\text { cept (5) }\end{array}$ & 2.5 & 2.8 & 2.8 \\
\hline $\begin{array}{l}\text { 19. Expected limit of systematic errors } \\
\text { from all sources except (5), including } \\
\text { sources not identified and treated here }\end{array}$ & 4.0 & 4.0 & 4.0 \\
\hline 20. Root-sum-square of (5) and (19) & 11. & 11. & 11. \\
\hline
\end{tabular}

\subsection{Wavelength of Light}

The light-scattering photometer employed for this work uses a mercury lamp in conjunction with a green filter in the incident beam which selectively transmits the so-called "mercury green line," and a similar filter in the scattered beam to remove any possible fluorescence from the solution. However, at the relatively high operating pressure of the mercury lamp, the green-line radiation is appreciably broadened. The light-scattering experiment senses the inverse fourth power average of the wavelength, as may be seen from eqs (5)-(10). Broadening of the radiation therefore produces an apparent shift in the effective wavelength. In addition, at sufficiently high pressures the center of the "green line" spectrum may shift. In order to estimate the size of the errors introduced into $M_{w}$ by these effects, we measured the combined optical transmission curve of the two green filters placed in the incident and scattered light paths. Using this transmission curve and a plot of the spectral distribution of lamp output supplied by the lamp manufacturer, we calculated the inverse-fourth power average wavelength of the scattered light reaching the detector, obtaining a result of $549.6 \mathrm{~nm}, 3.5 \mathrm{~nm}$ larger than the nominal value of $546.1 \mathrm{~nm}$ for the mercury green line. If the actual value of the inversefourth power average wavelength could be determined with sufficient accuracy, it could be used instead of the nominal value. However, the data on the spectral distribution of the lamp output are given to a resolution of only $5 \mathrm{~nm}$, and in addition are merely representative data for the type of lamp employed, rather than measured values for the lamps actually used. Direct measurement of the spectral distribution of the lamps used in this work was impractical, partly because of the relatively short lifetime of individual lamps, which resulted in several different lamps being used in the course of the measurements. We have therefore used the nominal value of the wavelength in our calculations, and have treated the deviation of $3.5 \mathrm{~nm}$ as a systematic error. In order to ascertain the effect of an error in wavelength of this size upon $M_{w}$, we first note from eqs (6) and (10) that the strongly wavelength-dependent quantities which appear in the calculation of $M_{w}$ are $\lambda_{0}$ itself and the index of refraction and Rayleigh ratio for benzene. From eq (10) and (11), the wavelength dependence of the calculated $M_{w}$ is that of the 
expression $\left(\lambda_{0}{ }^{4} U_{u} / n_{B}{ }^{2}\right)\left(2-\rho_{u}\right) /\left(1+\rho_{u}\right)$. Using the values reported by Coumou [3] for $U_{u}, n_{B}$, and $\rho_{u}$ at wavelengths of $436 \mathrm{~nm}$ and $546 \mathrm{~nm}$, we find that with increasing wavelength, $U_{u}$ decreases more strongly than $\lambda_{0}{ }^{-4}$, while $n_{B}$ and $\rho_{u}$ remain essentially constant. As a result, the combined expression increases by only 13 percent as the wavelength decreases from $546 \mathrm{~nm}$ to $436 \mathrm{~nm}$. Assuming a roughly linear dependence of the expression on wavelength, we find that a change of $3.5 \mathrm{~nm}$ corresponds to an error of 0.42 percent in $M_{w}$. Allowing for some nonlinearity and for the neglect of the wavelength dependence of $d n / d c$, we believe that 0.5 percent is a safe upper limit for systematic errors in $M_{w}$ arising from uncertainty in the wavelength of the light used.

\subsection{Rayleigh Ratio of Benzene}

As described in section 3.2 , the value of the "verticalvertical" Rayleigh ratio for benzene was calculated from published [3] values of $U_{u}$ and $\rho_{u}$, using eq (11). However, the quantities directly measured were not $U_{u}$ and $\rho_{u}$, but rather $U_{u}$ and $U_{v}$, the unpolarized scattering from vertically polarized light. Now $V_{v}$ is simply expressed in terms of $U_{u}$ and $U_{v}$ as:

$$
V_{v}=3 / 2 U_{V}-U_{u},
$$

and the standard deviation in $V_{v}$ resulting from known standard deviations in (independent) measurements of $U_{v}$ and $U_{u}$ may be estimated as the square root of the sum of the squares of the standard deviations in $U_{u}$ and the quantity $3 / 2 U_{v}$. The standard deviations in $U_{u}$ and $U_{v}$ are given by Coumou as 3 percent and "better than 2 percent," respectively. In order to convert the relative standard deviation in $U_{v}$ to an absolute standard deviation, we use eq (12) to backcalculate ${ }^{3 / 2} U_{v}$ from the published value of $U_{u}$ and the value of $V_{v}$ calculated from eq (11), obtaining finally an implied standard deviation in $V_{v}$ of $0.82 \times 10^{-6} \mathrm{~cm}^{-1}$, or 4.6 percent. From this estimate of standard deviation and the account of experimental techniques given in [3], we believe that 10 percent is a reasonable upper limit for systematic error in the value of $V_{v}$ for benzene, and therefore for errors in our values of $M_{w}$ from this source.

\subsection{The Ratio $\mathrm{I}_{G} / \mathrm{I}_{B}$}

Seven replicate measurements were made of the ratio $I_{G} / I_{B}$ of the scattering signal obtained with the glass working standard to that obtained with benzene, at a scattering angle of $90^{\circ}$. The resulting standard deviation of the mean value of the ratio was 0.11 percent. Using the Student $t$ factor for 6 degrees of freedom, we obtain 95 percent confidence limits of 0.3 percent. The value of the ratio for the glass rod employed was about 2.5, close enough to unity that both signals could be measured without changing gain settings. Thus the only further source of error in the ratio is in the non-linearity of the detection system. If we allow 0.3 percent for error from this source, then we obtain our combined estimate for errors in $I_{G} / I_{B}$ and $M_{w}$ from both sources as the square root of the sum of the squares of the individual error estimates, or 0.4 percent.

\subsection{Polarizer Errors}

The geometric factor $g$ in eq (4) is unity only if the vertically oriented polarizers in the incident and scattered beams transmit no horizontally polarized light at all. In the general case, we must consider both components of polarization in both the incident and scattered beams. Let $\zeta_{i}$ be the ratio of the transmission coefficients for horizontally and vertically polarized light for the polarizer in the incident beam, and let $\zeta_{s}$ be the corresponding ratio for the polarizer in the scattered beam. Then the observed scattering signal will be proportional to $V_{v}+\zeta_{s} H_{v}+\zeta_{i} V_{h}+\zeta_{i} \zeta_{s} H_{h}$, where $V$ and $H$ denote the vertically and horizontally polarized components, respectively, of the scattered radiation, and the subscripts $v$ and $h$ denote the vertically and horizontally polarized components, respectively, of the incident radiation. The ratio $H_{v} / V_{v}=V_{h} / V_{v}$ is the depolarization ratio $\rho_{v}$ for vertically polarized light, related to $\rho_{u}$ by $\rho_{v}=\rho_{u} /\left(2-\rho_{u}\right)$. By considering the geometry of the scattering system, we find [15] that the $H_{h}$ component of the scattering observed at an angle $\theta$ is the sum of two components, one independent of depolarization and proportional to $\cos ^{2} \theta$, the other arising from depolarized scattering and proportional to $\rho_{v} \sin ^{2} \theta$. The observed scattering signal is then proportional to

$$
1+\left(\zeta_{i}+\zeta_{s}\right) \rho_{v}+\zeta_{i} \zeta_{s}\left(\cos ^{2} \theta+\rho_{v} \sin ^{2} \theta\right) .
$$

The measured values of $\zeta_{i}$ and $\zeta_{s}$ for the polarizers in our instrument are $0.9 \times 10^{-3}$ and $1.0 \times 10^{-3}$, respectively, therefore the term in $\zeta_{i} \zeta_{s}$ is negligible. The estimated optical anisotropies of polyethylenes discussed in section $4.12 \mathrm{imply}$ values of $\rho_{v}$ less than $0.8 \times 10^{-3}$, so the term $\left(\zeta_{i}+\zeta_{s}\right) \rho_{v}$ can also be neglected for the polyethylene measurements. Finally, for the calibrating measurements on benzene we have $\rho_{u}=0.41$, which gives $\rho_{v}=0.26$. The error in the benzene measurements is therefore $(0.9+1.0) \times 0.26 \times$ $10^{-3}$, which is zero to the nearest 0.1 percent, and is so entered on line 7 of table 3 .

\subsection{The Ratios $S_{G}(\theta, c)$}

The measured values $S_{G}(\theta, c)$ of the scattering from polyethylene solutions and solvent relative to the scattering from the glass working standard are subject to errors arising from non-linearity of the detection system. However, scattering from solvent and from the most dilute solutions, to which the value of $M_{w}$ is most sensitive, were measured at the same gain settings as the glass working standard. Therefore, we believe that the estimated error of 0.3 percent used in section 4.5 is also adequate as an estimate of systematic error in $S_{G}(\theta, c)$.

\subsection{Solution Concentration}

As stated in section $2.1 \mathrm{~b}$, solutions were made up by weight. Concentrations $c$ were calculated from the relation:

$$
c=w \rho=w \rho_{0} /\left[1-w\left(1-\rho_{0} \bar{v}\right)\right],
$$

where $w, \rho, \rho_{0}$, and $\bar{v}$ are weight fraction, solution density, solvent density, and partial specific volume, respectively. Thus, errors in $c$ can arise from errors in $w, \rho_{0}$, and $\bar{v}$. However, the value of $M_{w}$ is unaffected by errors in $\bar{v}$. This can be seen by observing that the coefficient $C_{00}$ in eq (5), from which $M_{w}$ is calculated by eq (6), is the zero-angle, zero-concentration limit of the product of $c$ and a quantity 
which is a function $f$ of the scattering angle and measured scattering signals. As $c$ approaches zero, $\rho$ approaches the limiting value $\rho_{0}$, so that using the left-hand equality of eq (13), we can rewrite $C_{00}$ as $\rho_{0}$ times the zero-angle, zeroconcentration limit of the product $w f$. Thus errors in $\bar{v}$ will not affect the final value of $M_{w}$; errors in $\rho_{0}$ will produce relative errors of the same size in $M_{w}$. We believe that our value for solvent density is accurate to 0.2 percent, as shown on line 9 of table 3.

Solute and solvent weights were measured on semimicro balances accurate to $0.1 \mathrm{mg}$. The resulting errors in solvent weights are of the order of a few parts per million, and can be neglected. In order to estimate the effect of errors in solute weights on the values of $M_{w}$ obtained, a series of comparison calculations was carried out. For each SRM, one of the sets of measurements described in section 2.1c was chosen, and the value of $M_{w}$ obtained from this set was taken as a "reference" value. The value for each solute weight in turn was then increased by $0.1 \mathrm{mg}$ and the value of $M_{w}$ recalculated. The resulting percent changes in the reference values of $M_{w}$ are shown in table 4 , together with the sum of the absolute values of the individual changes, their algebraic sum, and the square root of the sum of their squares (rootsum-square). The sum of the absolute values represents the error if every weighing is in error by the maximum amount possible and in the direction which maximizes the resulting error in $M_{w}$. We reject this estimate as overly pessimistic. The absolute value of the algebraic sum would be the appropriate measure if all the weighings were in error by the same amount, and the root-sum-square would be appropriate if the individual errors were of random sign. Since both these possibilities seem physically plausible, we select as our error estimate the larger of the absolute value of the algebraic sum and the root-sum-square. As shown in table 4, this turns out to be the root-sum-square in all three cases. The corresponding values are shown on line 10 of table 3.

TABLE 4. Percent errors in weight-average molecular weight $M_{w}$ introduced by assumed errors of 0.1 mg in solute weights

\begin{tabular}{|c|c|c|c|}
\hline & SRM 1482 & SRM 1483 & SRM 1484 \\
\hline Number of solutions in reference subset & 7 & 5 & 7 \\
\hline $\begin{array}{l}\text { Percent changes in } \boldsymbol{M}_{w} \text { from assumed changes in solute } \\
\text { weight }\end{array}$ & $\begin{array}{rr}-0.30, & -0.09 \\
0.00, & 0.06 \\
0.04, & 0.02 \\
-0.04 & \end{array}$ & $\begin{array}{rr}0.38, & 0.10 \\
-0.06, & -0.08 \\
0.03 & \end{array}$ & $\begin{array}{ll}-1.06, & -0.49, \\
-0.11, & 0.19, \\
-0.22, & 0.21, \\
-0.08 & \end{array}$ \\
\hline Sum of absolute values of above & 0.55 & 0.65 & 2.36 \\
\hline Algebraic sum of above & -0.31 & 0.37 & -1.12 \\
\hline Root-sum-square of above & 0.32 & 0.41 & 1.23 \\
\hline
\end{tabular}

\subsection{Reflection Correction}

As a result of the reflection of light from the surface of the scattering cell, the signal observed at a scattering angle $\theta$ will include a component due to light scattered through an angle $\pi+\theta$. Several expressions have been given $[2 \mathrm{~b}, 7$, 16] for the magnitude of this effect, which depends upon the system geometry and upon the indices of refraction of the scattering solution, the scattering cell, and the surrounding medium. When the effect is large, for example when the surrounding medium is air, explicit correction must be made to the observed scattering signals. For the work reported here, the surrounding medium was a silicone oil with an index of refraction of about 1.5 , so that all three media have about the same index of refraction. In this case, the correction will be very small, and it will suffice to set an upper limit on the error introduced by its neglect.

The fraction $f_{r}$ of light reflected at a perpendicular interface between regions with index of refraction $n_{1}$ and $n_{2}$ is given by Fresnel's formula as $f_{r}=\left[\left(n_{1}-n_{2}\right) /\left(n_{1}+n_{2}\right)\right]^{2}$. In the present case, all the indices of refraction involved are about 1.5; the maximum difference between them is about 0.1 , giving a value of $f_{r}$ about 0.001 . The fraction of radiation scattered through $\pi+\theta$ appearing in the output at scattering angle $\theta$ is approximately [7] $2 f_{r}$, or about 0.002 . Suppose we wish to estimate an intercept $a$ in the equation $\gamma=a-b x$, where $y$ is inversely proportional to the intensity $I$ of the scattering signal and $x=\sin ^{2}(\theta / 2)$, but the intensities observed (denoted by primes) are linear combinations of the "true" intensities at $\theta$ and $\pi+\theta$ :

$$
\begin{aligned}
& I_{1}{ }^{\prime}=(1-\delta) I_{1}+\delta I_{2} \\
& I_{2}{ }^{\prime}=\delta I_{1}+(1-\delta) I_{2},
\end{aligned}
$$

where the subscripts 1 and 2 denote scattering angles $\theta$ and $\pi+\theta$, respectively. We wish to find the error in the intercept $a$, determined from measurements at two points $\left(x_{1}, y_{1}\right)$ and $\left(x_{2}, y_{2}\right)$, where $y_{i}=1 / I_{i}$, due to the use of $I_{1}{ }^{\prime}$ and $I_{2}{ }^{\prime}$ instead of $I_{1}$ and $I_{2}$. Clearly the size of the error depends upon the relative sizes of $I_{1}$ and $I_{2}$; if $I_{1}$ is equal to $I_{2}$ no amount of mixing will produce an error in the intercept. Here we are interested in the case where $I_{2}$ is only slightly smaller than $I_{1}$, and we write:

$$
I_{1}{ }^{\prime}=I_{2}{ }^{\prime}(1+\epsilon) .
$$

Solving for the apparent intercept in terms of $\left(x_{1}, y_{1}{ }^{\prime}\right)$ and $\left(x_{2}, y_{2}{ }^{\prime}\right)$ and using eqs (14) and (15) as appropriate, we find after some manipulation that to second order in $\delta$ and $\epsilon$, the relative error in the intercept is given by

$$
\delta \epsilon\left(x_{2}+x_{1}\right) /\left(x_{2}-x_{1}\right) .
$$

Taking the maximum and minimum scattering angles used for $x_{2}$ and $x_{1}$, we have $\left(x_{2}+x_{1}\right) /\left(x_{2}-x_{1}\right)=\sqrt{ } 2$. For SRM 1484, with the strongest angle-dependence of the three SRM's, the observed scattering signal at $135^{\circ}$ was about 6 percent larger than that at $45^{\circ}$. Then we have $\epsilon=0.06$, and the relative error in $M_{w}$ from neglect of the reflection correction is $0.002 \times 0.06 \times \sqrt{ } 2$, or about 0.02 percent for SRM 1484 and even less for the other two SRM's. To the nearest 0.1 percent, this is zero, and is so entered on line 11 of table 3 . 


\subsection{Instrumental Misalignment}

As described in section 2.1a, the optical alignment of the photometer was checked by observing that the scattering signals obtained from solvent at angles of 45 and $135^{\circ}$ differed by less than 1 percent. From consideration of the instrument geometry and the alignment procedure, it appears that the likeliest misalignment is a constant offset in the scattering angle. For the vertically polarized scattering of vertically polarized light from solvent, the signal observed at scattering angle $\theta$ should be proportional to $\csc \boldsymbol{\theta}$. It can easily be shown that in this case, a difference in scattering signal at 45 and $135^{\circ}$ of 1 percent could be produced by an offset in the scattering angle of only $0.3^{\circ}$, which is consistent with our estimate of the accuracy of the alignment. Using the notation of section 4.9 , we find that the relative error in the intercept $a$, and therefore in $M_{w}$, arising from equal and opposite relative errors $\delta / 2$ in $y_{1}$ and $y_{2}$ is given by

$$
\left(x_{1}+x_{2}+2 \mathrm{x}_{1} \mathrm{x}_{2} \mathrm{~b} / \mathrm{a}\right)(\delta / 2) /\left(\mathrm{x}_{2}-\mathrm{x}_{1}\right) .
$$

For $x_{1}$ and $x_{2}$ corresponding to scattering angles of 45 and $135^{\circ}$ respectively, this reduces to the simpler form

$$
\delta\left(1+\frac{1}{4} b / a\right) \sqrt{ } 2 .
$$

Thus the error increases with $b / a$, which is proportional to the mean-square radius. However, even for SMR 1484, the term $\frac{1}{4} b / a$ is only about 0.05 , and the error given by the above expression for $\delta=0.01$ is 0.7 percent for all three SRM's.

\subsection{Refraction Correction}

The observed scattering signal is proportional to both the scattering volume "seen" by the detector and the angular aperture of the detector optics. In general, both these quantities are functions of the index of refraction of the scattering liquid and of the system geometry. Equation (10) is written for the special case where the size and uniformity of the incident light beam are such that the incident intensity is constant over the region "seen" by the detector. For the instrument employed in this work, this condition is only approximately satisfied and eq (10) is not strictly valid. In principle, the factor $n_{B}{ }^{2}$ in eq (10) should be replaced by a function of $n_{B}$, the index of refraction $n_{S}$ of the solutions being measured, the system geometry, and integrals of intensity over various parts of the beam profile. However, for our present purpose it is neither practical nor necessary $t$ obtain the exact expression. It is shown elsewhere [17] that in the special case where the incident beam dimension is much smaller than the region "seen" by the detector, the shape of the beam profile is irrelevant and the correct expression is obtained by replacing $n_{B}{ }^{2}$ in eq (10) by $n_{S} n_{B}\left(n_{B}+z\right) /\left(n_{S}+z\right)$, where $z$ is a function of system geometry equal to about $1 / 9$ for our instrument. The correction factor to be applied to eq (10) in this case would then be just $\left(n_{S} / n_{B}\right)\left(n_{B}+z\right) /\left(n_{S}+z\right)$. This represents the limiting case; in our instrument the beam and the detector aperture are roughly the same size. The correction will then be smaller, and we can use the small-beam expression as an upper limit on the error in eq (10) from refraction effects. Taking $n_{B}=1.503$ and $n_{S}=1.586$, we find a correction factor of 1.004 , or a limiting error of 0.4 percent.

\subsection{Anisotropy of Polyethylene}

Equations (6) and (10) are derived on the assumption that the light scattered by the solute is not depolarized. When the solute is optically anisotropic, $M_{w}$ in eq (6) should be replaced by $M_{w}(1+\xi)$, where the correction term $\xi$ is given [18] by:

$$
\xi=(1 / 45)(1 / M)\left(\left\langle\gamma^{2}\right\rangle / M\right)\left[4 \pi n_{S} N_{A} /(d n / d c)\right]^{2},
$$

where $\left\langle\gamma^{2}\right\rangle$ is the mean-square optical anisotropy of the solute molecules. For polyethylene, $\left\langle\gamma^{2}\right\rangle$ is given approximately [19] as $267 \times 10^{-50} \mathrm{~cm}^{6}$ per $\mathrm{CH}_{2}$ group. The ratio $\left\langle\gamma^{2}\right\rangle / M$ is just $1 / 14$ of this, or $1.91 \times 10^{-49} \mathrm{~cm}^{6} / \mathrm{g}$. Using this value, and values already cited for the other quantities in eq (16), we find values for $\xi$ of $0.001,0.0005$, and 0.0001 for SRM's 1482, 1483, and 1484, respectively. The corresponding error estimates are shown on line 14 of table 3 , rounded to the nearest 0.1 percent.

\subsection{Temperature Dependence of Scattering of Glass}

Since the glass rod used as a working standard was compared with benzene at $23{ }^{\circ} \mathrm{C}$ and with the polyethylene solutions at $135{ }^{\circ} \mathrm{C}$, any temperature dependence in the scattering from the glass rod will give rise to errors in our values of $M_{w}$. It appears [20] that for the type of glass used for the working standard, the temperature-dependent scattering should amount to no more than 1 percent of the total scattering, and the temperature dependence of this part should be no stronger than the first power of the absolute temperature. The resulting maximum error, for comparisons between 23 and $135^{\circ} \mathrm{C}$, is 0.4 percent.

\subsection{Cutoff of Virial Expansion}

As described in section 3.2, virial coefficients beyond the third were not included in the analysis of the light-scattering data. At the solution concentrations employed in this work, their contribution should be negligible. Nevertheless, their neglect constitutes a source of systematic error, upon which we now attempt to set a limit. We first examine the relative sizes of the terms $C_{00}, C_{10} c$, and $C_{20} c^{2}$ in eq (5) at the maximum concentrations at which measurements were taken for each of the three SRM's. The relative sizes turn out to be $1.00: 0.52: 0.08$ for SRM $1482,1.00: 0.70: 0.15$ for SRM 1483, and $1.00: 0.74: 0.09$ for SRM 1484. The series all appear to be converging at a satisfactory rate. In addition, in each case the ratio of the third term to the second is appreciably smaller than the ratio of the second term to the first. Lacking any means of estimating the size of the fourth virial coefficient directly, it seems sufficiently cautious to suppose that the ratio of the fourth term to the third will be no larger than the ratio of the second term to the first, i.e., $C_{30} \leq C_{10} C_{20} / C_{00}$. In terms of the virial coefficients, this amounts to the assumption $A_{4} \leq 3 / 2 M_{w} A_{2} A_{3}$, using the relation $A_{4}=\frac{1}{4} K^{\prime} C_{30}$, the analogue of eqs. (8) and (9) for the fourth virial coefficient. The effect of a value of $C_{30}$ of this maximum size upon the value of $M_{w}$ was found by a technique similar to the method described in 4.8 for estimating the effects of errors in solute weights. For each 
SRM, a typical set of measurements was chosen as a reference set, and the corresponding value of $M_{w}$ taken as the reference value. An "error" term $C_{30} c^{3}$, with $C_{30}$ determined as described above, was then subtracted from each value of $c / I_{c}$ in the set, and the value of $M_{w}$ recalculated. The resulting changes of 0.7 percent, 1.4 percent, and 0.9 percent for SRM 1482, 1483, and 1484, respectively, are shown on line 16 of table 3.

\subsection{Summary}

Estimates of the contributions of individual sources to the overall systematic error in $M_{w}$ are summarized in table 3 . It will be seen that the uncertainty in the Rayleigh ratio for benzene, shown on line 5 of table 3 , completely overwhelms all the other uncertainties. Furthermore, it is based on literature values, which may be subject to future revision. Therefore, we first consider all the listed sources of error except the Rayleigh ratio of benzene. The sums and root-sumsquares of these are also shown in table 3. As discussed in 4.8 , we believe that the simple sum is an overly pessimistic estimate of error, and that the root-sum-square is more realistic. However, both to temper this judgment and to allow for other sources of error not considered here explicitly, we form our final, "best" estimates by increasing the root-sumsquares values of 2.5 to 3 percent in line 18 to 4 percent, as shown on line 19. Finally, we form the root-sum-square of this value with the uncertainty in the Rayleigh ratio for benzene to obtain final estimates for systematic errors from all sources of 11 percent, as shown on line 20 of table 3 and on the certificates for SRM's 1482, 1483, and 1484.

\section{References}

[1] Verdier, P. H., and Wagner, H. L., The characterization of linear polyethylene SRM's 1482, 1483, and 1484. I. Introduction, J. Res. Nat. Bur. Stand. (U.S.) 83, No. 2. (Mar.-Apr. 1978).

[2] See, for example: (a) Kerker, M., The Scattering of Light (Academic Press, New York, 1969); (b) Yamakawa, H., Modern Theory of Polymer Solutions (Harper \& Row, New York, 1971); (c) Utiyama, H., Calibration and Correction Factors, Chapter 4 in Light Scattering from Polymer Solutions. Ed. M. B. Huglin, pp. 61-88 (Academic Press, New York, 1972).

[3] Coumou, D. J., Apparatus for the measurement of light scattering in liquids; measurement of the Rayleigh factor of benzene and of some other pure liquids, J. Colloid Sci. 15, 408-417 (1960).

[4] Norberg, P. H., and Sundelö, L. A., A study of the purity of some polystyrene samples by means of refractive index increment measurements, together with a calibration of a differential refractometer, Makromol. Chem. 77, 77-89 (1964).

[5] Wagner, H. L., The characterization of linear polyethylene SRM 1475. VII. Differential refractive index of polyethylene solutions, J. Res. Nat. Bur. Stand. (U.S.) 76A, 151-155 (Mar.-Apr. 1972).

[6] Schneider, F., Schliephake, D., and Klimmek, A., Dichtetabelle für wässrige Saccharolösung, Zucher 16, 17 (1963), Sonderbeilage pp. $1-19$.

[7] Frolen, L. J., Ross, G. S., Wims, A. M., and Verdier, P. H., The characterization of linear polvethylene SRM 1475. VIII. Light scattering studies on polyethylenes in 1-Chloronaphthalene, J. Res. Nat. Bur. Stand. (U.S.) 76A, 156-160 (Mar.-Apr. 1972).

[8] Wagner, H. L., and Hoeve, C. A. J., Effect of molecular weight on the refractive increment of polyethylene and $n$-Alkanes, J. Polymer Sci. Part A-2 9, 1763-1776 (1971).

[9] Wagner, H. L., and Verdier, P. H., The characterization of linear polyethylene SRM's 1482, 1483, and 1484. II. Number-average molecular weights by membrane osmometry, J. Res. Nat. Bur. Stand. (U.S.) 83, No. 2, (Mar.-Apr. 1978).

[10] Brown, J. E., and Verdier, P. H., The characterization of linear polyethylene SRM 1475. IX. Number average molecular weight of fractions by membrane osmometry, J. Res. Nat. Bur. Stand. (U.S.) 76A, 161-163 (Mar.-Apr. 1972).

[11] Kokle, V., Billmeyer, F. W., Jr., Muus, L. T., and Newitt, E. J., The molecular structure of polvethylene. XI. Weight- and numberaverage molecular weights of selected samples, J. Polymer Sci. 62, 251-261 (1962).

[12] Chiang, R., Intrinsic viscosity-molecular weight relationship for fractions of linear polyethylene, J. Phys. Chem. 69, 1645-1653 (1965).

[13] See, for example, Flory, P. J., Principles of Polymer Chemistry, (Cornell University Press, Ithaca, New York, 1953); Morawetz, H., Macromolecules in Solution (Interscience Publishers, New York 1965).

[14] See, for example, Handbook of Mathematical Functions, M. Abramowitz, and I. A. Stegun, Ed., Nat. Bur. Stand. (U.S.), Appl. Math. Ser. 55 (1964).

[15] Reference 2a, Chapter 10.

[16] Kratohvil, J. P., Calibration of light-scattering instruments. IV. Corrections for reflection effects, J. Coll. Interface Sci. 21, 498$512(1966)$.

[17] Verdier, P. H., to be published.

[18] Utiyama, H., and Tsunashima, Y., Measurements of anisotropic light scattering and thermal transition in optical anisotropy of poly- $\alpha$ methylstyrene, J. Chem. Phys. 56, 1626-1633 (1972).

[19] Jernigan, R. L., and Flory, P. J., Optical anisotropy of chain molecules. Theory of depolarization of scattered light with application to $n$-Alkanes, J. Chem. Phys. 47, 1999-2007 (1967).

[20] Macedo, P. B., private communication. 